\title{
Editorial
}
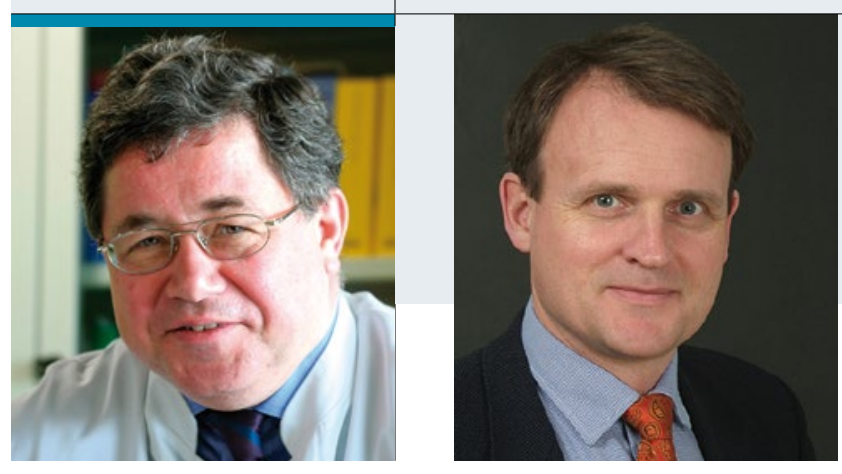

"Selbst barrierefördernde Wirkungen

der Vaseline lassen sich auf

AhR-Liganden zurückführen."

Prof. Dr. Hans F. Merk, Hautklinik der Medizinischen Fakultät, Universitätsklinikum der RWTH Aachen

Prof. Dr. Thilo Jakob, Klinik für Dermatologie und Allergologie,

Universitätsklinikum Gießen und Marburg, Standort Gießen

\section{Allergologie in Tiefe und Breite}

A llergien sind umweltbedingte Erkrankungen par excellence. Es ist daher kein Wunder, dass ein zellulärer Sensor für zahlreiche Umweltsubstanzen wie der Arylhydrocarbon-Rezeptor $(\mathrm{AhR})$ auch vielfältige pathophysiologische Beziehungen zu allergischen und Autoimmunerkrankungen aufweist. Diesem Rezeptor war Anfang August ein dreitägiges Symposium in Rochester (New York) gewidmet. Bislang ist der AhR häufig als ein Sensor zur Vermittlung toxischer Eigenschaften von 2,3,7,8-Tetrachlorodibenzo-p-dioxin (TCDD) aufgefasst worden. Es stellte sich jedoch heraus, dass er auf molekularer Ebene als Liganden-aktivierter Transkriptionsfaktor in viele zelluläre Prozesse eingreift, zudem zahlreiche verschiedene exogene $\mathrm{Li}$ ganden aber auch endogene Liganden wie Tryptophan-Metabolite hat, die ihn zu einem zentralen Sensor der Zellen für Umwelteinflüsse macht. Er wirkt nicht nur auf immunkompetente Zellen wie antigenpräsentierende dendritische Zellen und TLymphozyten ein, sondern nimmt auch direkten Einfluss auf die gestörte Barrierebildung der Haut bei atopischer Dermatitis. Schon früh wurde gezeigt, dass steinkohlenteerhaltige Externa AhR-abhängige Enzymaktivitäten wie Cytochrom $\mathrm{P} 450$ (1A1/1A2/1B1) induzieren und damit solche Arzneimittel AhR als primäres Zielprotein haben. Das konnte inzwischen bestätigt werden und selbst barrierefördernde Wirkungen der Vaseline lassen sich auf AhR-Liganden zurückführen. Zudem hemmt eine Aktivierung des AhR auch Entzündungsreaktionen der Haut durch AhR-sensitive T-Lymphozytensubpopulationen. Es ist daher nicht erstaunlich, dass inzwischen nach AhR-Liganden gesucht wird, die als neue Therapeutika bei Allergien und chronisch-entzündlichen Hauterkrankungen eingesetzt werden können. Einen ausführlichen Bericht und Literaturhinweise finden Sie in dieser Ausgabe auf S. 82.

Während es sich bei diesem Symposium um ein thematisch sehr fokussiertes Thema handelte, erscheint diese Ausgabe des Allergo Journal zum Deutschen Allergie Kongress in Berlin, der das gesamte Gebiet der Allergien aktuell präsentiert. Nunmehr zum 11. Mal werden alle allergologisch interessierten Kollegen der Arbeitsmedizin, Dermatologie, HNO, Pädiatrie, Pneumologie und anderer Gebiete mit Interesse an der Thematik „Moderne Allergologie - Konzepte für die Zukunft" zusammengeführt. Besonderer Dank gilt den Kongresspräsidenten Prof. Susanne Lau und PD Dr. Jörg Kleine-Tebbe, denen es zusammen mit dem wissenschaftlichen Beirat gelungen ist, auch in einer die Organisation nicht immer erleichternden Umwelt ein spannendes und für alle Allergologen ansprechendes Programm zu gestalten. Diese Ausgabe des Allergo Journal begleitet den Kongress mit aktuellen Übersichten zu Entwicklungen der spezifischen Immuntherapie mit Peptiden, epigenetischen Einflüssen auf die Pathogenese allergischer Erkrankungen und neuen diagnostischen Hinweisen zum Beispiel bei Alveolitiden. Zudem finden Sie ab S. 50 die Kongressabstracts. $\mathrm{Zu}$ Kongress und Studium dieses Heftes wünschen wir Ihnen neue interessante Erkenntnisse.

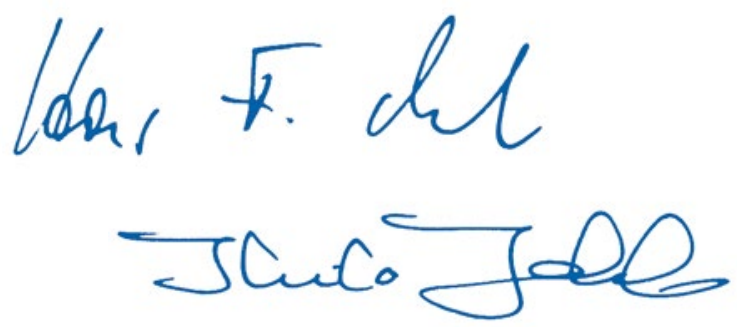

